\title{
Case report of a patient studied by brain SPECT and several neuropsychological tests for assessing correlation between drugs abuse, cognitive impairment and onset of psychotic disorder
}

\author{
Giuseppe Miti ${ }^{1}$, Adriana Borriello ${ }^{1}$, Rosa Bandiera ${ }^{1}$, Emanuela Falconi ${ }^{2}$ and Lucio Mango ${ }^{3 *}$ \\ ${ }^{1}$ Department of Psychiatry, "San Camillo-Forlanini” General Hospital - Rome, Italy \\ ${ }^{2}$ Addiction service (Ser.D), ASL Rm6(5th district) - Velletri (Rome), Italy \\ ${ }^{3}$ Department of Nuclear Medicine, "San Camillo-Forlanini” General Hospital - Rome, Italy
}

\begin{abstract}
Is reported here a case of young patient with prevalent previous diagnosis of personality disorder, submitted to several neuropsychological tests and perfusion brain SPECT, in order to assess the relationship between substance abuse, the results of brain SPECT, done at the onset of symptoms of the psychiatric disorder, and an assessment of cognitive abilities. In particular, tests were used to assess cognitive flexibility, working memory, attention, short and long-term memory. This case shows the presence of a link between cognitive deficits, hypoperfusion in specific areas of the brain revealed by SPECT and the evolution of diagnosis from axis II to axis I, according to DSM IV-TR.
\end{abstract}

\section{Introduction}

Substance abuse is quite prevalent in adolescents in metropolitan cities and is often characterized by the simultaneous abuse of several substances. Moreover, faster and faster are daily produced new synthetic substances that give rise to abuse and of which we do not know the long-term effects and for which have not yet been produced reagents to track them down in the human body [1].

It is now widespread belief that most of the substances of abuse can result in psychological, behavioral and psychiatric consequences. In fact, even if a single substance may not have high neurotoxicity characteristics, the simultaneous use of more than one substance makes the long-term effect highly likely. The old theory that saw the use of substances such as an attempt to self-care of previous psychiatric or subthreshold problems, currently is no longer considered likely, thanks to the knowledge of the neurotoxic effects, mechanisms of action, negative consequences of taking in psychotic patients, and in light of recent research in brain imaging.

MRI in subjects with chronic methamphetamine abnormalities reported severe abnormalities of limbic, paralimbic and cingulate cortex, white hypertrophy and decreased hippocampal volume and consequent cognitive deficits, particularly the memory system [2].

As well known, SPECT allows to determine the regional concentration of the perfusion tracer [3]. SPECT is widely available and can assess perfusion in brain tissue and the localization and extent of lesions as a result of defects in blood supply can be assessed by SPECT studies [4]. In schizophrenic patients has been found in numerous studies a reduction of regional cerebral blood flow (rCBF), mainly involving the frontal lobes [5]. In a recent study, some authors showed that amphetamine can induce diffuse and non-homogeneous disorders of brain perfusion which was more prominent in the right hemisphere [6].

Studies performed with brain SPECT in patients who had chronic abuse by solvent inhalation showed a statistically significant reduction in blood flow in the prefrontal brain districts, bilaterally [7]. The hypoperfusion severity was correlated to the degree of severity of the volition and apathy of the SANS scale, suggesting an association with the amotivational syndrome. This finding appears to be an anomaly in the early stage of the disease and not secondary to neuroleptic treatment, having been found in both treatment-naive and in treatment-free subjects. On the symptomatic piano, the finding of a reduction of perfusion at the frontal site was correlated with the presence of symptoms mainly negative. While a reduction of $\mathrm{rCBF}$ on temporal site was demonstrated in both untreated patients and in patients undergoing pharmacological therapy with neuroleptics, and seems to be correlated with the severity of positive symptoms.

In order to, correlate qualitatively and semi-quantitatively the presence of areas of impaired cerebral perfusion and areas of cortical atrophy with the presence of neurocognitive alterations, we studied a cohort of patients [8], from which we extrapolated the one presented here.

Correspondence to: Prof. Lucio Mango, Direttore Servizio di Medicina Nucleare, A.O. "S.Camillo-Forlanini”, Piazza Carlo Forlanini, 1 - 00151 Roma, Italy, Tel: +390655552541; Fax: +390655552442; E-mail:1.mango@scf.gov.it

Key words: substances abuse, psychiatric disorders, perfusional brain SPECT

Received: August 05, 2017; Accepted: August 22, 2017; Published: August 25, 2017 
Mango L (2017) Case report of a patient studied by brain SPECT and several neuropsychological tests for assessing correlation between drugs abuse, cognitive impairment and onset of psychotic disorder

\section{Case presentation}

Is reported here the case of a young patient ( $25 \mathrm{yrs})$ come to our observation for substances abuse (cannabinoids and ecstasy) since when he was $18 \mathrm{yrs}$ old and antisocial personality disorder.

After an accurate medical history to assess familiarity of psychiatric disorders, history of substance abuse, symptomatic onset and psychiatric symptoms possibly present, to the patient the following neuropsychological tests were administered:

1. Digit Span (Forward-Backward)

2. Color-Word Stroop Test

3. Serial Repeat of Bisexual Words

4. Spinnler's Attentive Matrices (Visual Search)

5. Rey Auditory List Learning Test

6. Colored Progressive Matrices Raven's (CPM)

7. F.A.S. (Verbal Fluency Task)

8. Wisconsin Card Sorting Test (WCST)

The response to those tests showed a mild psychotic involvement. It is well known that the Wisconsin Card Sorting Task (WCST) has been used to assess dysfunction of the prefrontal cortex and basal ganglia, as demonstrated by some authors, by means of event-related functional magnetic resonance imaging (fMRI) to study the pattern of activation during four distinct stages in the performance of this task, resulting in specific involvement of different prefrontal areas during different stages of task performance [9].

The patient was subsequently subjected to perfusion brain SPECT. Twenty minutes after accessing IV line and in quiet environment, 740
MBq of ${ }^{99 \mathrm{~m}} \mathrm{Tc}-\mathrm{ECD}$ was administered and after 40 minutes of radiotracer injection, the SPECT images were obtained. Brain perfusion scans were interpreted by two nuclear medicine specialists separately which were blind; if contradictory results were obtained viewpoint of third nuclear medicine specialist would be exerted. Images obtained (Figure1), showed a decrease of the perfusion in left frontal and temporo-mesial areas, a distribution compatible with a schizophrenia situation.

\section{Discussion}

The case presented here appears worthy of interest because of the different results arising from cognitive tests, which showed a mild involvement in particular of the frontal lobes, and SPECT, on the contrary strongly pathologic with aspects related to schizophrenia.

Given the importance of early intervention, and the lack of effective treatment options, there is a clear need for effective, scalable treatments [10]. Recent reports [11,12] have shown that motivation plays a significant and mediating role between neurocognition, social cognition and functional outcome. Preliminary data shown by some authors, seem clearly to emphasize the clinical relevance of an early cognitive assessment and of a Cognitive Remediation intervention too, applied as early as possible (within the "critical period") to young psychotic onset, particularly regarding the possibility to affect the executive functions [13].

The use of a Cognitive Protocol facilitates the measurement of the increase and decrease of deficits over time both in terms of therapeutic decisions and the course of the disease. It is also useful to evaluate both cognitive and standard rehabilitation outcomes, enabling to verify the results of the integrated psychological therapy. This technique involves a series of exercises related not only to the cognitive functions but also to social and personal problems solving skills [14].

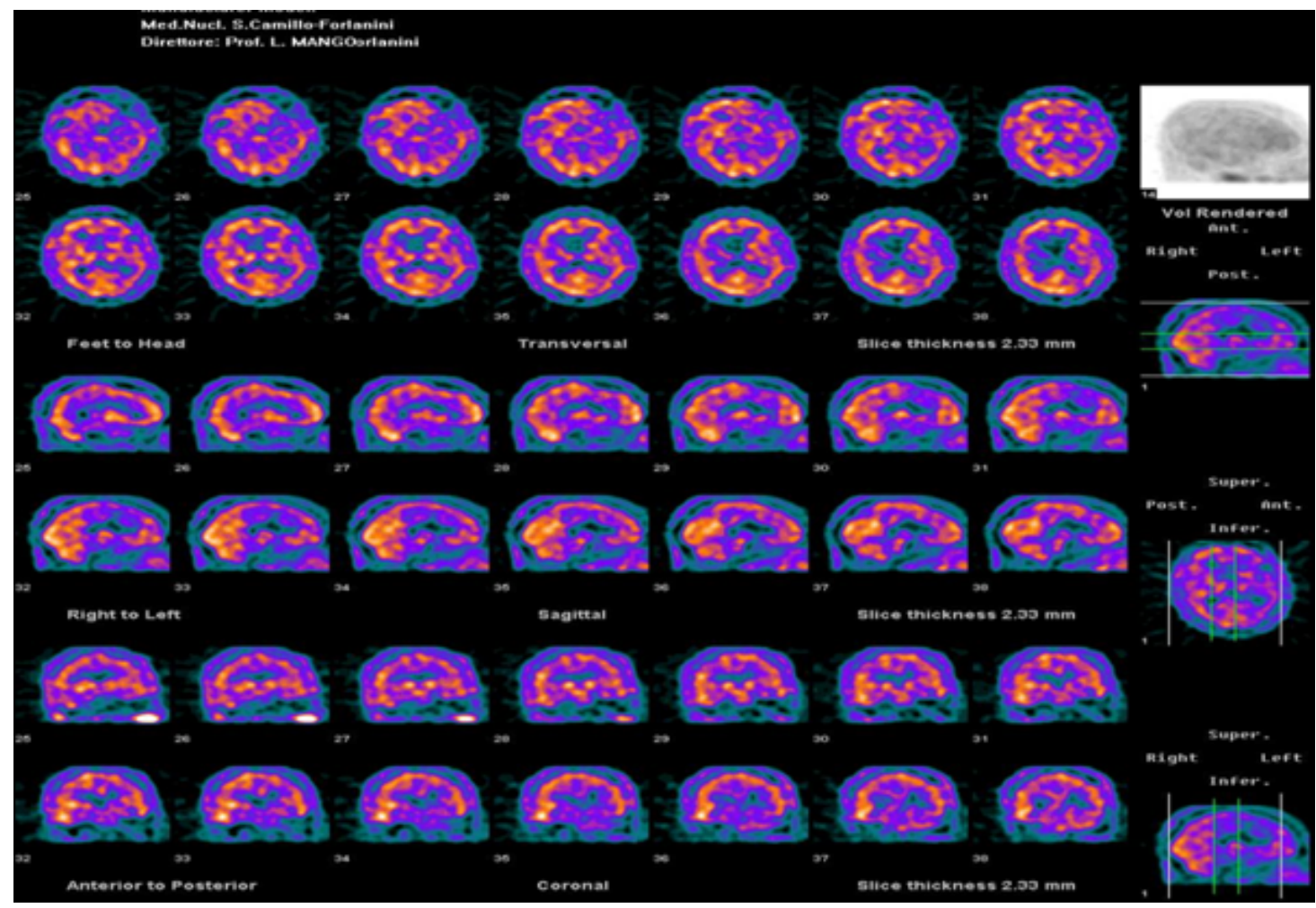

Figure 1. Perfusional brain SPECT showing significant activity reduction in left frontal lobe and left temporo-mesial area. 
Mango L (2017) Case report of a patient studied by brain SPECT and several neuropsychological tests for assessing correlation between drugs abuse, cognitive impairment and onset of psychotic disorder

In conclusion, our objective is to carry out a retest of the neuropsychological assessment and a cerebral SPECT after a predefined time period. Data so obtained will be compared with results derived from a group of patients who performed a cognitive rehabilitation through the Integrated Psychological Therapy (IPT) and with those obtained by a group that did not.

\section{References}

1. ESPAD Report 2015 (2016) Results from the European School Survey Project on Alcohol and Other Drugs. European Monitoring Centre on Drugs and Drug Addiction.

2. Thompson PM, Hayashi KM, Simon SL, Geaga JA, Hong MS, et al. (2004) Structural abnormalities in the brains of human subjects who use methamphetamine. J Neurosci 24: 6028-6036. [Crossref]

3. Kuhl DE, Edwards RQ, Ricci AR, Yacob RJ, Mich TJ, et al. (1976) The Mark IV system for radionuclide computed tomography of the brain. Radiology 121: 405-413. [Crossref]

4. Heiss WD (2014) Radionuclide imaging in ischemic stroke. J Nucl Med 55: 1831-1841. [Crossref]

5. Faget-Agius C, Boyer L, Richieri R, Auquier P, Lançon C, et al. (2016) Functional brain substrate of quality of life in patients with schizophrenia: A brain SPECT multidimensional analysis. Psychiatry Res 249: 67-75. [Crossref]

6. Vahid R, Dabbagh K, Fereshteh MS, Bita D (2017) Abnormal cerebral blood flow in methamphetamine abusers assessed by brain perfusion single emission computed tomography. Iran J Nucl Med 25: 47-51.
7. Küçük NO, Kiliç EO, Ibis E, Aysev A, Gençoglu EA, et al. (2000) Brain SPECT findings in long-term inhalant abuse. Nucl Med Commun 21: 769-773. [Crossref]

8. Miti G, Borriello A, Bandiera R, Mango L (2014) Correlation between drugs abuse, cognitive impairment and onset of a psychotic disorder: a pilot study using SPECT and neuropsychological assessment. III International Conference on NOVEL PSYCHOACTIVE SUBSTANCES (NPS), Rome (Italy).

9. Monchi O, Petrides M, Petre V, Worsley K, Dagher A (2001) Wisconsin card sorting revisited: Distinct neural circuits participating in different stages of the task identified by event- related functional magnetic resonance imaging. $J$ Neurosci 21: 7733-7741. [Crossref]

10. Nahum M, Fisher M, Loewy R, Poelke G, Ventura J, et al (2014) A novel, online social cognitive training program for young adults with schizophrenia: A pilot study. Schizophr Res Cogn 1: e11-e19. [Crossref]

11. Gard DE, Fisher M, Garrett C, Genevsky A, Vinogradov S (2009) Motivation and its relationship to neurocognition, social cognition, and functional outcome in schizophrenia. Schizophr Res 115: 74-81. [Crossref]

12. Green MF, Hellemann G, Horan WP, Lee J, Wynn JK (2012) From perception to functional outcome in schizophrenia: modeling the role of ability and motivation. Arch Gen Psychiatry 69: 1216-1224. [Crossref]

13. Borriello A, Balbi A, Menichincheri RM, Mirabella F (2015) Timing and effectivenes of Brenner's IPT cognitive training in early psychosis. A pilot study. Riv Psichiatr 50: 127-133. [Crossref]

14. Borriello A, Dionisi A, Iannucci C, Balbi A (2002) Operative criteria for the measurement of schizophrenic patient cognitive deficits within a public operated psichiatric environment. Riv Psichiatr 37: 20-28.

Copyright: (C2017 Mango L. This is an open-access article distributed under the terms of the Creative Commons Attribution License, which permits unrestricted use, distribution, and reproduction in any medium, provided the original author and source are credited. 